\title{
Vapor Sensing Using Conjugated Molecule-Linked Au Nanoparticles in a Silica Matrix
}

\author{
Shawn M. Dirk, ${ }^{1}$ Stephen W. Howell, ${ }^{2}$ B. Katherine Price, ${ }^{3}$ Hongyou Fan, ${ }^{4}$ Cody Washburn, ${ }^{1}$ \\ David R. Wheeler, ${ }^{5}$ James M. Tour, ${ }^{3}$ Joshua Whiting, ${ }^{6}$ and R. Joseph Simonson ${ }^{6}$
}

${ }^{1}$ Organic Materials Department, Sandia National Laboratories, MS-0892, Albuquerque, NM 87185, USA

${ }^{2}$ Advanced Sensor Technologies Department, Sandia National Laboratories, MS-0892, Albuquerque, NM 87185, USA

${ }^{3}$ Departments of Chemistry and Mechanical Engineering and Materials Science, The Smalley Institute for

Nanoscale Science and Technology, Rice University, MS-222, 6100, Houston, TX 77005, USA

${ }^{4}$ Ceramic Processing and Inorganic Department, Sandia National Laboratories, MS-1349, Albuquerque, NM 87185, USA

${ }^{5}$ Biosensors and Nanomaterials Department, Sandia National Laboratories, MS-0892, Albuquerque, NM 87185, USA

${ }^{6}$ Micro-Total-Analytical Systems Department, Sandia National Laboratories, MS-0892, Albuquerque, NM 87185, USA

Correspondence should be addressed to Shawn M. Dirk, smdirk@sandia.gov

Received 20 June 2008; Accepted 10 October 2008

Recommended by Jose A. Pomposo

\begin{abstract}
Cross-linked assemblies of nanoparticles are of great value as chemiresistor-type sensors. Herein, we report a simple method to fabricate a chemiresistor-type sensor that minimizes the swelling transduction mechanism while optimizing the change in dielectric response. Sensors prepared with this methodology showed enhanced chemoselectivity for phosphonates which are useful surrogates for chemical weapons. Chemoselective sensors were fabricated using an aqueous solution of gold nanoparticles that were then cross-linked in the presence of the silica precursor, tetraethyl orthosilicate with the $\alpha$-, $\omega$-dithiolate (which is derived from the in situ deprotection of 1,4-di(Phenylethynyl-4', $4^{\prime \prime}$-diacetylthio)-benzene (1) with wet triethylamine). The cross-linked nanoparticles and silica matrix were drop coated onto interdigitated electrodes having $8 \mu \mathrm{m}$ spacing. Samples were exposed to a series of analytes including dimethyl methylphosphonate (DMMP), octane, and toluene. A limit of detection was obtained for each analyte. Sensors assembled in this fashion were more sensitive to dimethyl methylphosphonate than to octane by a factor of 1000 .
\end{abstract}

Copyright (c) 2009 Shawn M. Dirk et al. This is an open access article distributed under the Creative Commons Attribution License, which permits unrestricted use, distribution, and reproduction in any medium, provided the original work is properly cited.

\section{Introduction}

The ability to effectively detect harmful chemical agents, including chemical weapons, is a huge concern in today's world. Numerous types of chemicals can be used as chemical warfare agents, each with widely different structures, making detection of them increasingly difficult. One approach to chemical detection involves the use of sensors comprised of conductive elements including carbon particles [1], carbon nanotubes [2-4], and metal nanoparticles [5-14], each embedded in a nonconductive matrix applied to a set of interdigitated electrodes (IDEs) to provide sensors known as chemiresistors. In the above cases, the nonconductive matrix imparts chemoselectivity. Typically in metal nanoparticlebased chemiresistors, this matrix is the coating on metal nanoparticles.
Chemiresistors may be probed by evaluating the current passing though the sensor as a bias is applied to one of the IDEs. Several publications have described the signal transduction mechanism as an activated tunneling model that contains two terms as shown in the following equation $[10,15,16]$ :

$$
\sigma=\sigma_{0} \mathrm{e}^{(-\delta \beta)} \mathrm{e}^{(-\mathrm{E} / \mathrm{RT})}
$$

In this expression, $\sigma$ is the electronic conductivity of the film, $\delta$ is the interparticle distance, $\beta$ is the electronic coupling coefficient (effectively a measure of the density of states (DOS) available between conducting particles), Ea is the activation energy for electron transfer between adjacent conducting particles, $R$ is the gas constant, and $T$ is the absolute temperature. The first exponential factor takes into 
account the effect of nanoparticle spacing and the DOS overlaps between nanoparticles, and the second exponential term is related to the permittivity of the film.

In most cases, the analyte affects both factors of the activated tunneling model serving both as a solvent, swelling the nanoparticle/matrix, as well as changing the effective dielectric constant of the sensing film. In the case of low dielectric constant analytes, the first exponential factor dominates, and conductivity decreases. In the case of analytes with high dielectric constants, the second factor dominates, and conductivity increases in the presence of the analyte.

In this article, we report the assembly of amine-capped nanoparticles in the presence of both thiolate cross-linker and silica precursor. This nanoparticle cross-linker silica assembly was then deposited onto interdigitated electrodes and exposed to a series of analyte vapors in varying concentrations. Selectivity is provided by both the cross-linking ligand and the silica matrix surrounding the nanoparticles. The silica precursor was chosen to minimize the contribution of the first exponential factor of the activated tunneling mechanism by effectively fixing the value of $\delta$, that is, by creating a relatively rigid matrix which exhibits little swelling. This allows the activated tunneling change upon exposure to be dominated by the second factor in order to increase the sensitivity of the sensor to high dielectric constant $(k)$ compounds like dimethyl methylphosphonate DMMP $(k=$ 22.3) [17].

\section{Experimental Details}

2.1. Materials and Methods. All reactions were carried out under a dry nitrogen atmosphere. ${ }^{1} \mathrm{H}$ NMR spectra were obtained on a $400 \mathrm{MHz}$ Bruker (Fremont, Calif, USA) Avance-400. Proton chemical shifts $(\delta)$ are reported relative to tetramethylsilane (TMS). UV-vis measurements were performed on a Perkin-Elmer (Waltham, Mass, USA) Lambda 25. Centrifugation was performed using a Fisher (Suwanee, Calif, USA) Scientific Marathon $8 \mathrm{~K}$ centrifuge. Hydrogen tetrachloroaurate (p.a.), tetraoctylammonium bromide (98\%), cetyl trimethyl ammonium bromide (99+\%), 1,4diiodobenzene (98\%), trimethylsilylacetylene (98\%), dodecylamine (98\%), dichlorodimethylsilane (99\%), tetraethyl orthosilicate (98\%), and sulfuric acid (reagent grade) were purchased and used as received from Acros. (Geel, Belgium) Sodium borohydride (99\%), potassium carbonate (99\%), tris(dibenzylideneacetone)dipalladium(0), copper(I) iodide (99.999\%), triphenylphosphine (99\%), tetrahydrofuran (anhydrous, 99.9\%, inhibitor free), 1,2-dichloroethane (99\%), and hydrogen peroxide (30\% solution in water, ACS Reagent) were purchased and used as received from Aldrich. (Milwaukee, Wis, USA) Toluene (certified ACS), methylene chloride (HPLC grade), chloroform (HPLC grade), carbon disulfide (reagent grade), dimethylacetamide (certified), and hexanes (HPLC grade) were purchased from Fisher (Suwanee, Calif, USA) and used as received. Triethylamine (TEA, reagent grade) was purchased from Fisher (Suwanee, Calif, USA) and vacuum was transferred over calcium hydride prior to use in coupling reactions. 4-Iodobenzenesulphonyl chloride (95\%) was purchased and used as received from Lancaster (Windham, NH, USA).

\subsection{Preparation of Au Nanoparticles}

2.2.1. Thiol-Capped Au Nanoparticles. The preparation of the capped gold nanoparticles followed the Brust method [18]. The synthesis of gold nanoparticles involved the transfer of hydrogen tetrachloroaurate $(0.35 \mathrm{~g}, 0.87 \mathrm{mmol})$ dissolved in water $(25 \mathrm{~mL})$ to a solution of tetraoctylammonium bromide $(2.19 \mathrm{~g}, 4.01 \mathrm{mmol})$ in toluene $(80 \mathrm{~mL})$. After the gold salt transferred into the organic phase, the aqueous phase was discarded. Octanethiol $(26 \mu \mathrm{L}, 0.15 \mathrm{mmol})$ was added, and sodium borohydride $(0.38 \mathrm{~g}, 10.0 \mathrm{mmol})$ in water $(25 \mathrm{~mL})$ was added too. The solution turned dark red almost immediately, and after 15 minutes the organic phase was separated and passed through a $0.45 \mu \mathrm{m}$ Teflon filter. The organic solvent was almost completely removed by rotary evaporation, and the resulting solid material was dissolved in a minimal amount of toluene $(\sim 3 \mathrm{~mL})$ followed by precipitation with ethanol. The ethanolic suspension was subjected to centrifugation (2000 rpm for 10 minutes) to collect the nanoparticles after decanting the supernate. The precipitation procedure was repeated, and the nanoparticles were stored at $-20^{\circ} \mathrm{C}$.

2.2.2. Amine-Capped Au Nanoparticles. The preparation followed a modified Brust method [18]. The dodecylamine replaced the thiol component. Tetrachloroaurate (112 mg) was dissolved with water $(25 \mathrm{~mL})$ and poured into a $500 \mathrm{~mL}$ round bottomed flask. Tetraoctylammonium bromide $(0.37 \mathrm{~g}, 0.68 \mathrm{mmol})$ was dissolved into toluene $(25 \mathrm{~mL})$ and added to the round bottom flask with stirring. Once the aqueous layer was clear, a solution of dodecylamine $(574 \mathrm{mg}$ in toluene, $25 \mathrm{~mL}$ ) capping agent was added. A fresh solution of sodium borohydride ( $165 \mathrm{mg}$ in water, $25 \mathrm{~mL}$ ) was added, and the color immediately changed to very dark red. The contents were stirred for 1 hour. The layers were separated, and the aqueous layer was discarded. The organic solvent was almost completely removed by rotary evaporation. The solid material was dissolved in a minimal amount of toluene $(\sim 2 \mathrm{~mL})$ and then precipitated with ethanol. The suspension was subjected to centrifugation (2000 rpm for 10 minutes) to collect the nanoparticles, and the decanted liquid was discarded. The precipitation procedure was repeated, and the nanoparticles were stored at $-20^{\circ} \mathrm{C}$.

2.2.3. Transfer of Au Nanoparticles into Water [19]. Au nanoparticles $(100 \mu \mathrm{g})$ dissolved in chloroform $(3 \mathrm{~mL})$ were added to a $100 \mathrm{~mL}$ Erlenmeyer flask. A solution of cetyl trimethyl ammonium bromide $(20 \mu \mathrm{g})$ dissolved in water $(10 \mathrm{~mL})$ was also added to the Erlenmeyer flask. The twophase mixture was heated until all the chloroform was removed, and the nanoparticles were transferred to the aqueous phase. The nanoparticles were characterized using TEM after the transfer was complete. The nanoparticles had an average size range of 4-6 nm (radius for several batches). 


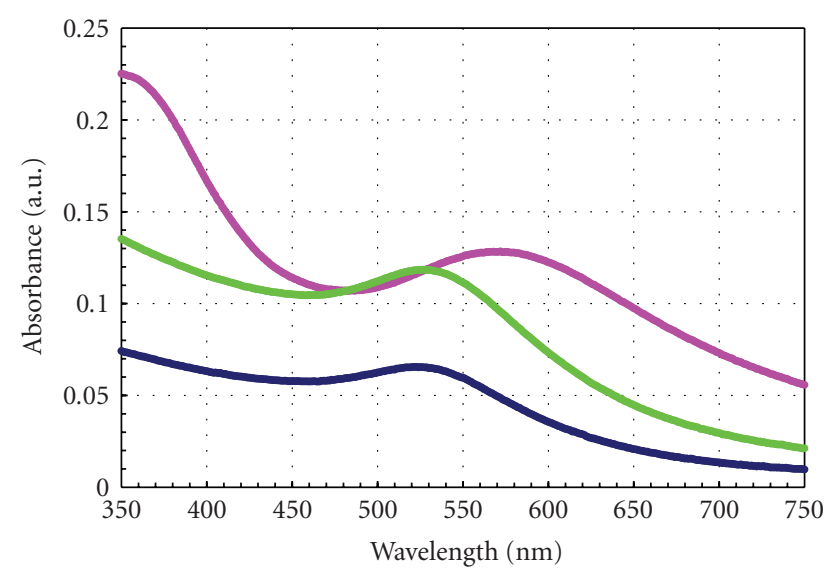

- Au and TEA

- Au, TEA, and 1

- Au only

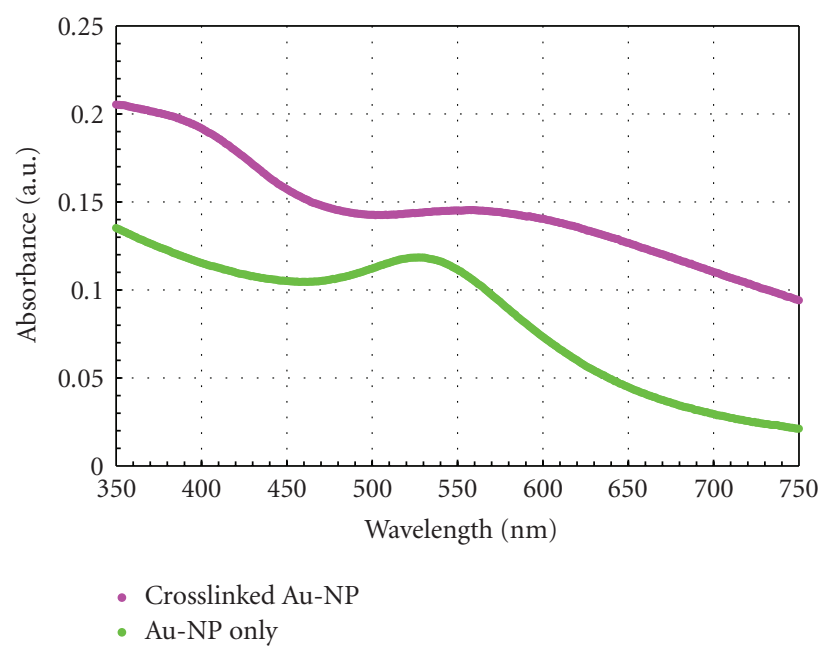

(b)

FIGURE 1: UV-vis spectra of (a) alkyl amine capped NPs only and crosslinked, and (b) alkanethiol-capped NPs only and the 1-crosslinked $\mathrm{Au}-\mathrm{NP}$. The shifts are 45 and $34 \mathrm{~nm}$, respectively.

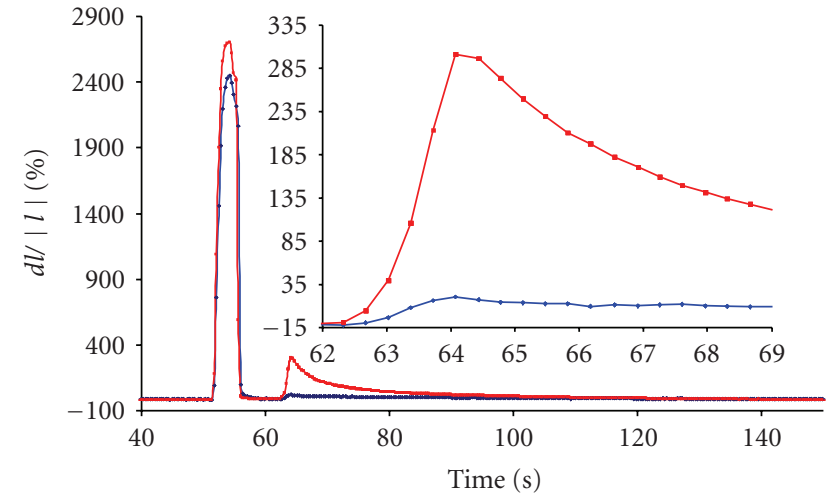

Figure 2: $1000 \mathrm{ppm}$ DMMP exposure $(1000 \mathrm{ppm} \max )$ to TEOS + NP (blue line) and TEOS + PE + NP films (red line). The inset shows an expansion of the DMMP peak.

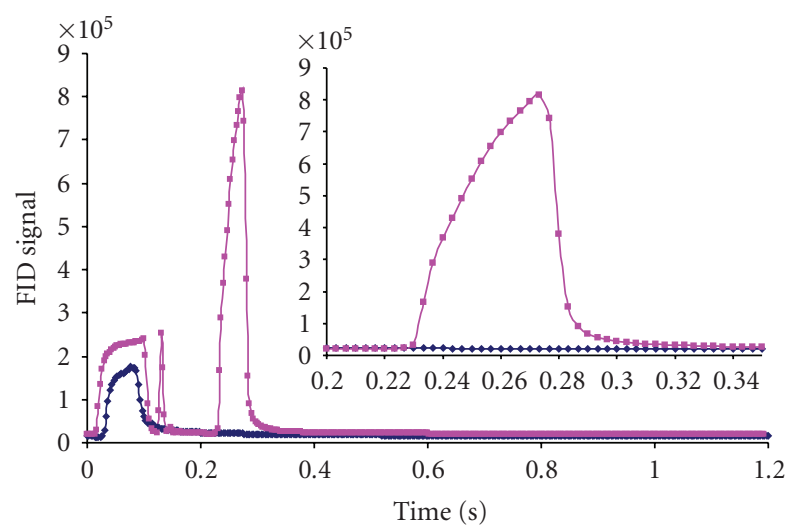

FIGURE 3: The response of the FID detector to a $1000 \mathrm{ppm}$ DMMP in $\mathrm{CS}_{2}$ injection with $(\boldsymbol{\square})$, and without a TEOS + NP + PE ( $)$ film before the FID detector. The inset shows an expansion of the DMMP peak.

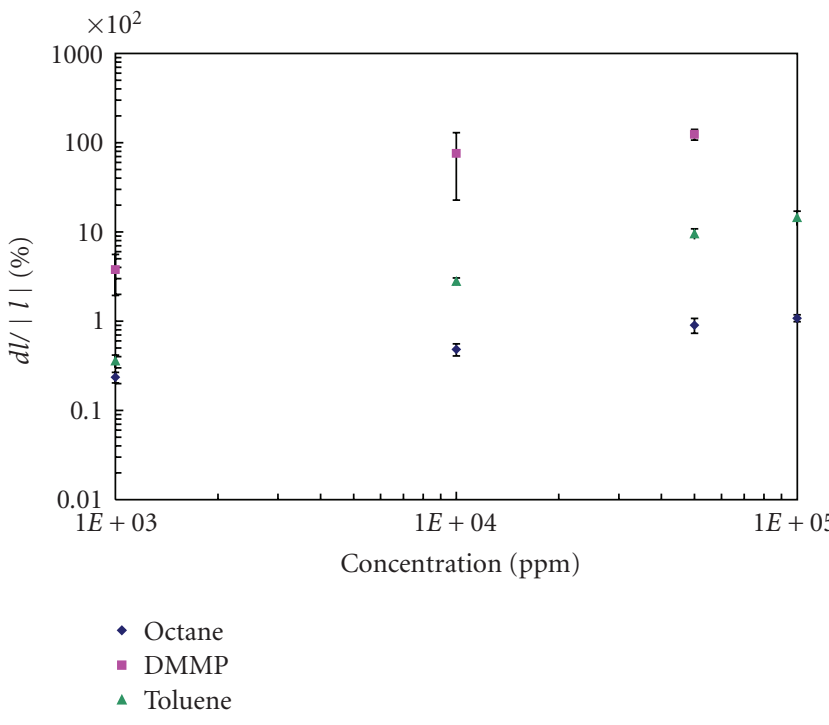

FIGURE 4: Comparison of the response of films consisting of $\mathrm{TEOS}+\mathrm{PE}+\mathrm{NP}$ when exposed to octane $(\boldsymbol{\vee})$, toluene $(\boldsymbol{\Delta})$, and DMMP $(\boldsymbol{\square})$. The response of the film is presented as a $\log / \log$ plot for clarity.

\subsection{Preparation of Oligophenylene Ethynylene}

2.3.1. General Pd/Cu Coupling Reaction Procedures [20]. All solids, including the aryl halide, alkyne, copper iodide, triphenylphosphine, and palladium catalyst, were added to an oven dried sealed glass tube. The atmosphere was removed via vacuum and replaced with dry argon. THF, remaining liquids, and triethylamine were added during stirring. The reaction was then heated if required. Upon cooling, the reaction mixture was filtered via gravity filtration to remove the solids and then diluted with methylene chloride. The 


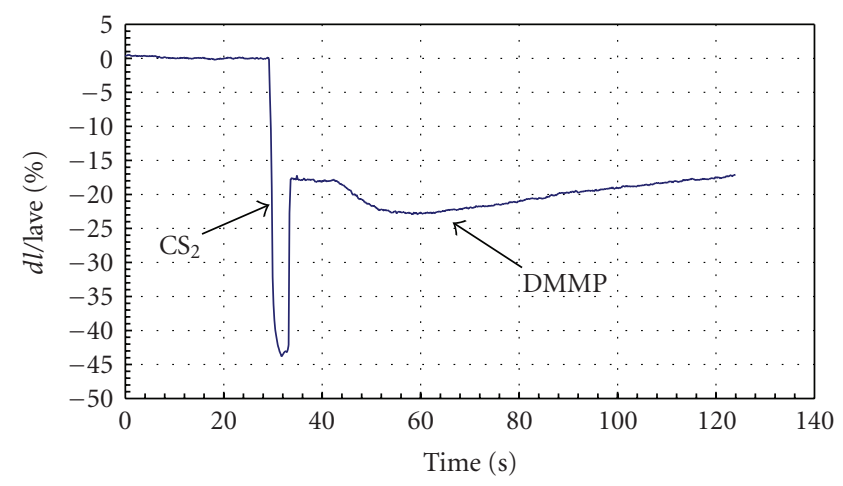

(a)

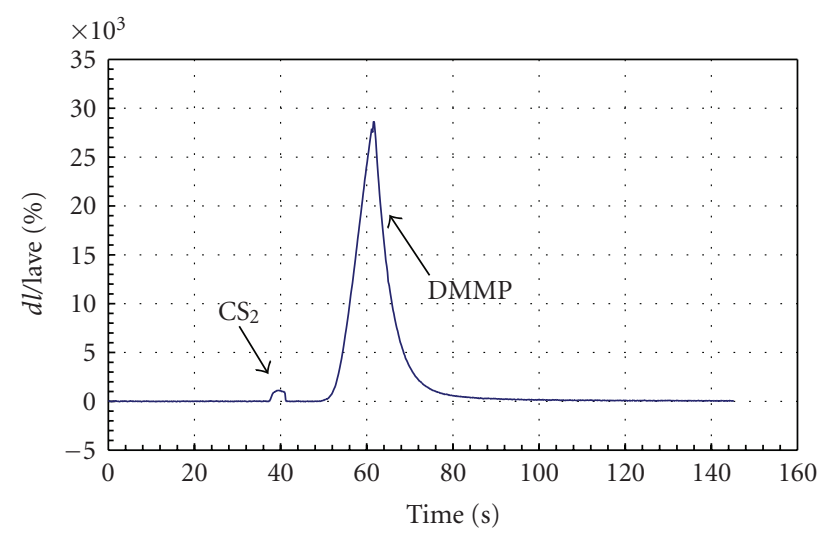

(c)

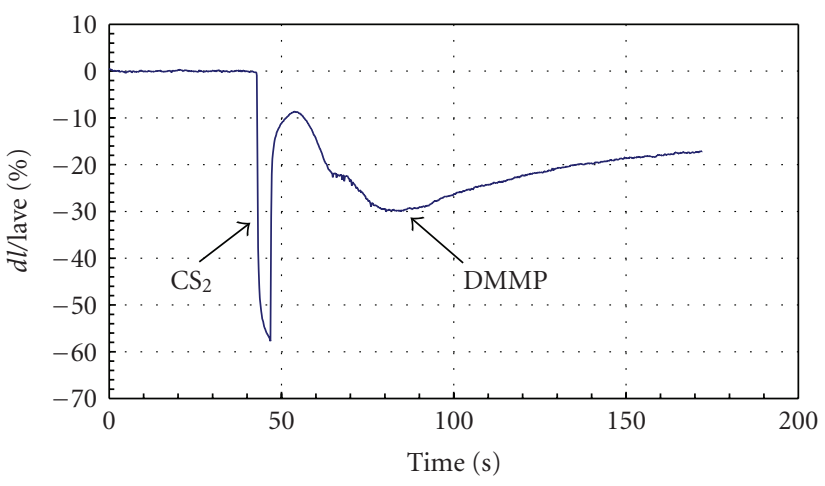

(b)

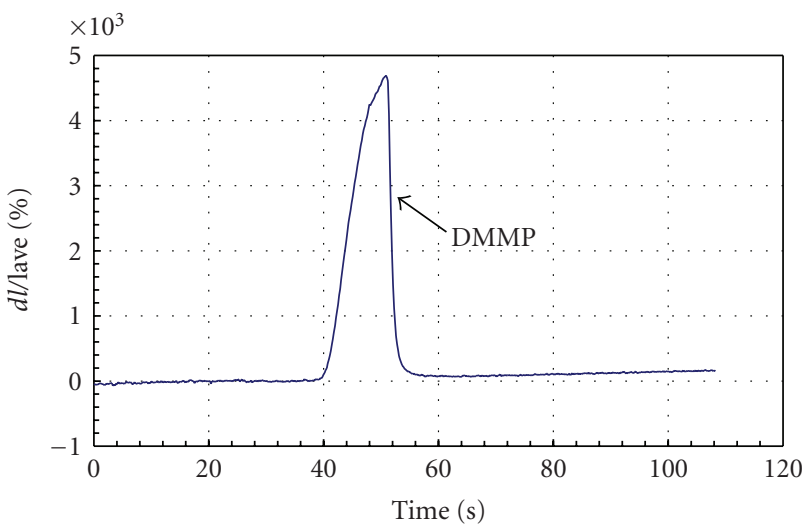

(d)

FIgURE 5: Response of gold nanoparticle/10 mL TEOS coated IDEs when exposed to $10000 \mathrm{ppm}$ DMMP in CS 2 . The amount of Au nanoparticles was decreased while keeping the TEOS amount fixed. (a) contained $1.25 \mathrm{mg}$ Au NP:10 uL TEOS, (b) contained 0.63 mg Au NP:10 uL TEOS, (c) contained 0.31 mg Au NP:10 uL TEOS, (d) contained 0.16 mg Au NP:10 uL TEOS.

reaction mixture was extracted with aqueous ammonium chloride $(\times 3)$. The organic layer was dried with magnesium sulfate and then filtered, and the solvent was removed via rotary evaporation.

\subsubsection{General Procedure for the Deprotection of Trimethylsilyl-} Protected Alkynes [21]. The protected alkyne, potassium carbonate (5 equivalents per protected alkyne), methanol, and methylene chloride were added to a round bottom flask equipped with a stir bar. The reaction was stirred at room temperature, and upon completion the reaction mixture was diluted with methylene chloride and washed with brine $(3 \times)$. The organic layer was dried over anhydrous magnesium sulfate, and the solvent was removed via rotary evaporation.

2.3.3. Synthesis of 1,4-Bis(Trimethylsilylethynyl)Benzene [22]. Diiodobenzene ( $3.30 \mathrm{~g}, 10 \mathrm{mmol})$, tris(dibenzylidenacetone) dipalladium $(0)(0.28 \mathrm{~g}, 0.30 \mathrm{mmol})$, copper(I) iodide $(0.23 \mathrm{~g}$, $1.20 \mathrm{mmol})$, triphenylphosphine $(0.63 \mathrm{~g}, 2.40 \mathrm{mmol})$, trimethylsilyl acetylene $(3.2 \mathrm{~mL}, 22.0 \mathrm{mmol})$, triethylamine $(5.6 \mathrm{~mL})$, and tetrahydrofuran $(20 \mathrm{~mL})$ were coupled according to the general coupling procedure above. The crude product was carried directly onto the next synthetic step.
2.3.4. Synthesis of 1,4(Diethynyl)Benzene [22]. 1,4-Bis(trimethylsilylethynyl)benzene was deprotected with potassium carbonate $(8.3 \mathrm{~g}, 60.0 \mathrm{mmol})$, methanol $(50 \mathrm{~mL})$, and dichloromethane $(50 \mathrm{~mL})$ according to the general deprotection procedure above to yield $1.5 \mathrm{~g}(100 \%)$ of a crystalline pale white solid, ${ }^{1} \mathrm{H}$ NMR (400 MHz, $\left.\mathrm{CDCl}_{3}\right) 7.42$ (s, 4H), $3.15(\mathrm{~s}, 2 \mathrm{H})$.

2.3.5. Synthesis of S-Acetyl-4-Iodothiophenol [23]. 4-iodobenzenesulphonyl chloride $(6.1 \mathrm{~g}, 20.0 \mathrm{mmol})$ was added to a $250 \mathrm{~mL}$ round bottom flask, and the atmosphere was removed via vacuum and replaced with argon. Zinc powder $(4.6 \mathrm{~g}, 70.0 \mathrm{mmol})$ was added to a $500 \mathrm{~mL}$ three-neck round bottom flask equipped with a stir bar and an addition funnel, and the atmosphere was removed via vacuum and replaced with argon. Dimethylacetamide $(5.6 \mathrm{~mL}, 60.0 \mathrm{mmol})$ and 1,2-dichloroethane $(100 \mathrm{~mL})$ were added to the $250 \mathrm{~mL}$ round bottom flask, and the contents were transferred via cannula into the addition funnel. 1,2-dichloroethane $(100 \mathrm{~mL})$ and dimethyldichlorosilane were added to the $500 \mathrm{~mL}$ three-neck flask $(8.5 \mathrm{~mL}, 70.0 \mathrm{mmol})$. The contents of the addition funnel were added drop-wise over a period of 30 minutes to the $500 \mathrm{~mL}$ flask. After the complete addition, the reaction was heated to $75^{\circ} \mathrm{C}$ for 2 hours. The reaction 
mixture was cooled to room temperature, and potassium carbonate $(1.52 \mathrm{~g}, 11.0 \mathrm{mmol})$ and acetyl chloride $(5.7 \mathrm{~mL}$, $80.0 \mathrm{mmol}$ ) were added to the reaction flask; the contents were stirred overnight. The crude reaction was worked up with brine and extracted with dichloromethane. The organic phase was dried over magnesium sulfate and then filtered, and the solvents were removed via rotary evaporation to yield $5.4 \mathrm{~g}(97 \%)$ of a crystalline white solid, ${ }^{1} \mathrm{H}$ NMR $(400 \mathrm{MHz}$, $\left.\mathrm{CDCl}_{3}\right) 7.72(d t, J=8.4,2 \mathrm{~Hz}, 2 \mathrm{H}), 7.11(d t, J=8.4 \mathrm{~Hz}$, $2.4 \mathrm{~Hz}, 2 \mathrm{H}) 2.41(\mathrm{~s}, 3 \mathrm{H})$.

Synthesis of 1,4-di(Phenylethynyl-4', $4^{\prime \prime}$-Diacetylthio)Benzene (1) [24]. S-Acetyl-4-iodothiophenol (2.78 g, 10.0 $\mathrm{mmol}), \quad 1,4$ (diethynyl)benzene $(0.63 \mathrm{~g}, 5.0 \mathrm{mmol})$, tris (dibenzylideneacetone)dipalladium(0) $(0.14 \mathrm{~g}, 0.15 \mathrm{mmol})$, copper iodide $(0.11 \mathrm{~g}, 0.60 \mathrm{mmol})$, triphenylphosphine $(0.31 \mathrm{~g}, \quad 1.20 \mathrm{mmol})$, triethylamine $(5.6 \mathrm{~mL})$, and tetrahydrofuran $(20 \mathrm{~mL})$ were coupled according to the general coupling procedure above. The crude product was purified via column chromatography (1:1 hexanes:dichloromethane) to yield $1.21 \mathrm{~g}(82 \%)$ of a pale brown solid, ${ }^{1} \mathrm{H}$ NMR $\left(400 \mathrm{MHz}, \mathrm{CDCl}_{3}\right) 7.61(\mathrm{~m}, 8 \mathrm{H}) 7.46$ $(d, J=8.4 \mathrm{~Hz}, 4 \mathrm{H}) 2.45(\mathrm{~s}, 6 \mathrm{H})$.

2.4. Cross-Linking the Au Nanoparticles. An aqueous solution of Au nanoparticles $(0.5 \mathrm{~mL})$ and $\mathbf{1}(100 \mu \mathrm{L}, 1 \mathrm{mmol}$ in THF) were combined. Triethylamine $(5 \mu \mathrm{L})$ was then added, and a color change was evident within 5 minutes. Tetraethyl orthosilicate (TEOS, $10 \mu \mathrm{L}$ ) was then added and agitated. UV-vis measurements were taken within 5 minutes after the TEA and TEOS additions to confirm cross-linking.

2.5. Cross-Linked Nanoparticle Film Deposition. Interdigitated electrode devices were cleaned with acetone (30 seconds bath and rinse) and rinsed with DI water. The device was then submerged in Piranha (1:1 30\% hydrogen peroxide and conc. sulfuric acid) for 2 minutes and rinsed with copious amounts of DI water. Caution: piranha is a very strong oxidizer and reacts violently with organics. The surface was dried with $\mathrm{CO}_{2}$. Prior to Au nanoparticle/silica deposition, the interdigitated electrode containing substrate was electrically characterized as open using a digital multimeter. The films were produced by flooding the surface with the cross-linked $\mathrm{Au}$ nanoparticle/silica solution and allowing the solvent to evaporate. The films that were produced in this manner had reproducible initial resistances. The thickness was measured with atomic force microscopy (AFM).

2.6. Substrate Exposure to Analytes. Dimethyl methylphosphonate, toluene, and octane were evaluated as analytes. The analytes are dissolved in $1 \mathrm{~mL} \mathrm{CS}$ and injected into a Hewlett Packard 5850 split injection gas chromatograph equipped with 1 meter $100 \mu \mathrm{m}$ ID capillary column coated with a polydimethylsiloxane stationary phase at ambient temperature. The carrier gas was hydrogen, and the split was 0.33 . The flow rate through the column was $28 \mathrm{sccm}$. The injection port was heated to $250^{\circ} \mathrm{C}$. Gas flow exited the capillary column and entered a custom-made test fixture described below. Gas left the test fixture and returned to the HP FID detector heated to $250^{\circ} \mathrm{C}$. The injection volume was $1 \mu \mathrm{L}$, and repeated injections were performed with an autoinjection tower for reproducibility.

After the Au nanoparticle/silica assembly, the functionalized substrate was placed in a custom-made silco-coated stainless steel test fixture where the assembled substrate sits in a recessed slot. The lid of the test fixture contained pogo pins that were connected to a Keithley 6487 current preamp voltage source. The lid included a milled channel to allow gas flow over the $8 \mu \mathrm{m}$ interdigitated electrodes. The lid was sealed with an external O-ring. The voltage source and current measurement instruments were controlled by a custom LabVIEW program. Prior to analyte exposure, an $\mathrm{I}(\mathrm{V})$ trace was performed by cycling the voltage from $0 \mathrm{mV}$ to $100 \mathrm{mV}$ to $-100 \mathrm{mV}$ to $0 \mathrm{mV}$ in order to determine the resistance of the working sensor device. During an analyte exposure, $100 \mathrm{mV}$ was typically applied to the substrate, and the current was measured.

\section{Results and Discussion}

3.1. Preparation of the Nanoparticle/Silica Film. Initially Brust-type [18] nanoparticles capped with alkyl thiols were transferred into water by the method of Fan and used to create a nanoparticle film. The strategy employed was to mix the nanoparticles, a silica precursor (tetraethyl orthosilicate), and a cross-linking molecule $\mathbf{1}$ together in the presence of a reagent to facilitate both the hydrolysis of the tetraethyl orthosilicate and the deprotection of the thioacetate groups as shown in Scheme 1.

Two common methods used to deprotect thiol acetate groups include $\mathrm{NH}_{4} \mathrm{OH}$ in THF [25] and $\mathrm{H}_{2} \mathrm{SO}_{4}$ in $\mathrm{CH}_{2} \mathrm{Cl}_{2} / \mathrm{CH}_{3} \mathrm{OH}$ [26]. In our case, we were limited to water as the solvent, and attempts to deprotect the thiol acetate groups with these protocols in the presence of the silica precursor tetraethyl orthosilicate and the amine-capped $\mathrm{Au}$ nanoparticles proved unsuccessful. Attempted ammonium hydroxide which facilitated deprotection of the thioacetate group resulted in a very fast TEOS condensation rate, and a silica gel resulted within seconds of the base addition. This gellation precluded the desired organic cross-linking of the metal nanoparticles. In the case of the $\mathrm{H}_{2} \mathrm{SO}_{4}$ facilitated deprotection, the rate of condensation of the hydrolyzed tetraethyl orthosilicate was slower than the basecatalyzed case; however, the deprotection of the thioacetate in water was still slower, so that adequate cross-linking or the nanoparticles were again precluded (as determined by NMR).

Sharporenko et al. successfully demonstrated that a thiol acetate group could be deprotected with wet triethylamine in situ to form a self-assembled monolayer [27]. The use of this deprotection method resulted in a useable ratio of the TEOS condensation rate to thiol acetate deprotection rate in the work described here. A color change was apparent within minutes, confirming cross-linking of the Au nanoparticles (NPs) due to the expected change in plasmon resonance. If the solution was allowed to react (cross-link) for several more minutes ( $\sim 10$ minutes), the nanoparticles precipitated from 


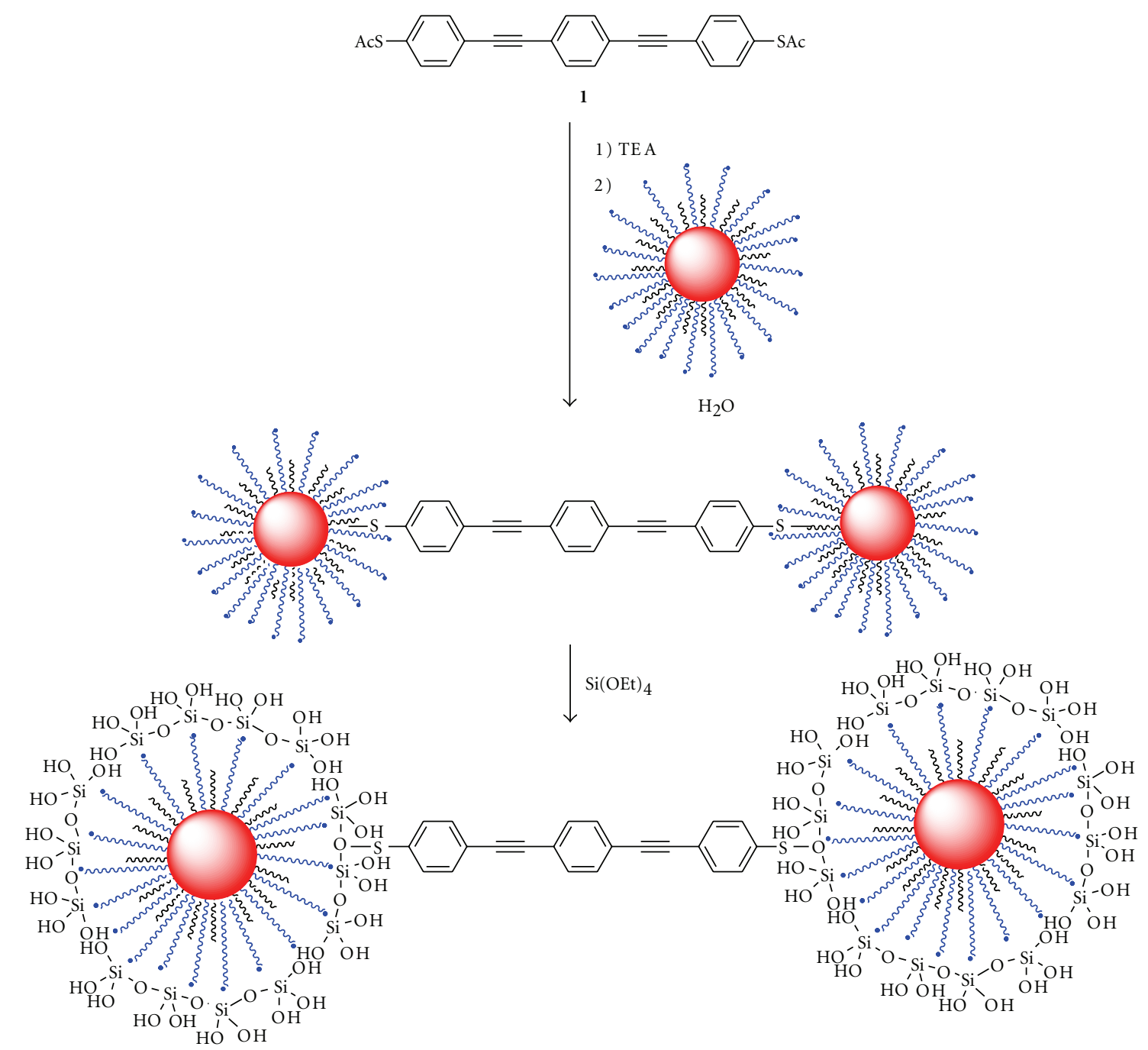

Scheme 1: Shows the assembly process used to form phenylene ethynylene crosslinked Au nanoparticles in a silica matrix.

solution. This color change was confirmed and measured with the use of UV-visible (UV-vis) absorption spectroscopy of the nanoparticles in aqueous solution. Absorption spectra of the NPs were acquired throughout the cross-linking process to observe changes in the plasmon resonance. A shift correlates to a change in resonance attributed to the crosslinking of the NPs. These shifts are observed in the spectra displayed in Figure 1.

We found that dodecylamine-capped Au nanoparticles were much easier to cross-link (and gave a larger red shift in UV-vis) than the corresponding thiol-capped $\mathrm{Au}$ nanoparticles, presumably due to faster ligand exchange kinetics. Dodecylamine-capped Au nanoparticles were prepared according to the method of Brust [18] except that dodecylamine was used in place of the alkanethiol capping agent as shown in Scheme 2.

Cross-linked Au/silica films of dodecylamine-capped nanoparticles were prepared by drop-casting onto Piranhacleaned $8 \mu \mathrm{m}$ Au interdigitated electrodes after a period of 5 minutes from the addition of triethylamine. The solvent was allowed to evaporate and air dry for a period of no less than 3 hours. The drop cast films were characterized using AFM to measure the thickness of the assembled film. The typical film thickness was determined to be $25 \mathrm{~nm}$.

\subsection{The Use of the Dodecylamine-Capped Nanoparticle/Silica} Films as Sensor Elements. The coated substrate was mounted into a test fixture that was placed inside a GC equipped with a flame ionization detector (FID) in line with the column (see Scheme 3). The device was exposed to various concentrations of DMMP, toluene, and octane dissolved in carbon disulfide.

Figure 2 shows a typical plot of the normalized (The plot was normalized by dividing the change in current upon exposure to analyte by the initial current.) change in current, as a 1000-ppm-DMMP (904 ppm maximum solution concentration $(\mathrm{v} / \mathrm{v})$, not taking the GC split into account) sample is injected onto the column. The first peak observed is the $\mathrm{CS}_{2}$ solvent peak followed by a second slightly 


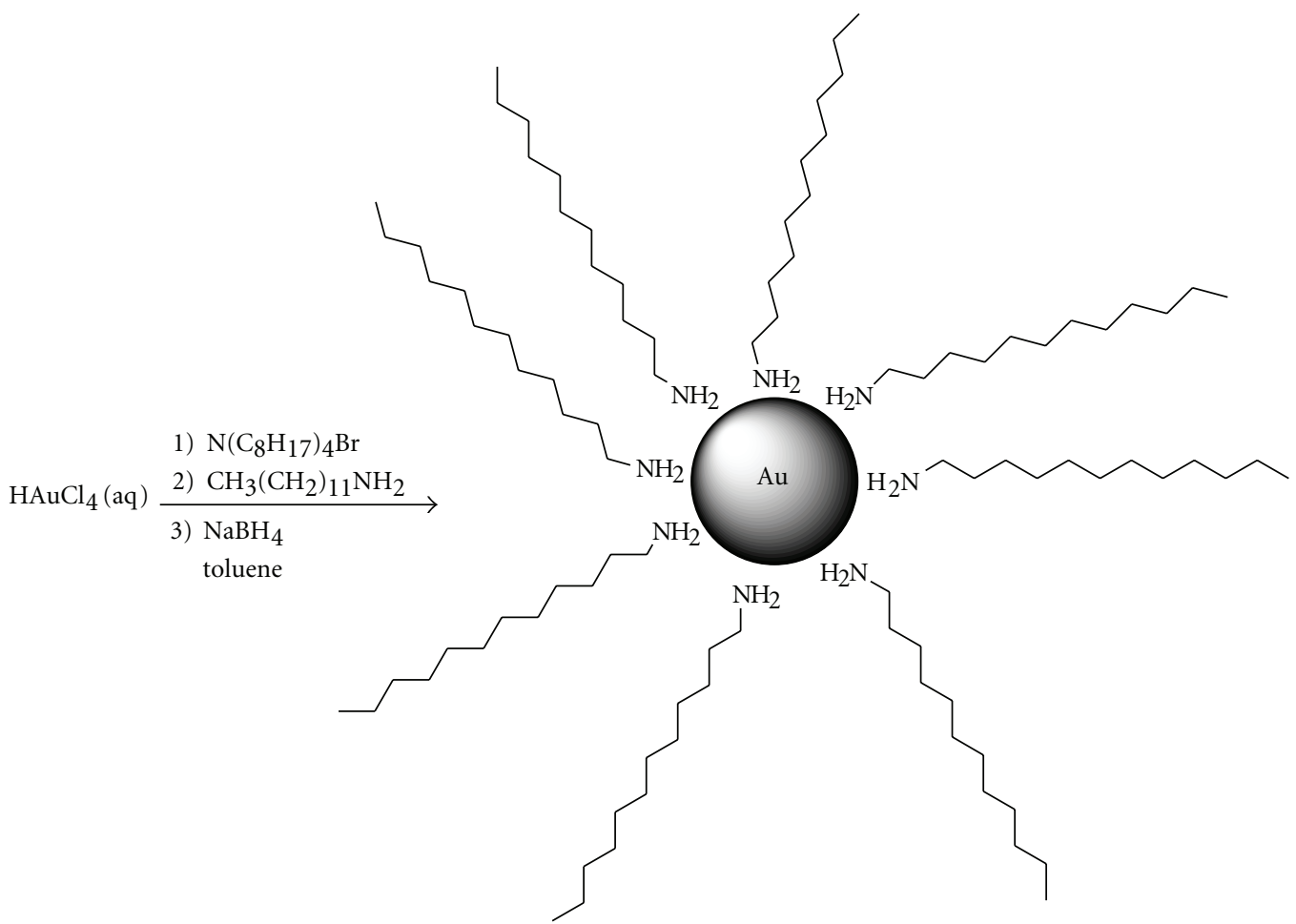

Scheme 2: Preparation of alkyl amine gold nanoparticles by a modification of the Brust method [18].

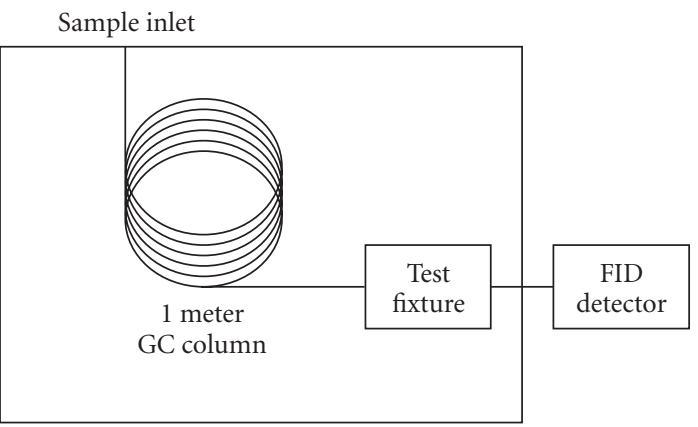

HP 5850 GC oven

Scheme 3: The layout of the test structure used to determine analyte selectivity and sensitivity.

broader DMMP peak. The blue trace is the normalized change in signal from an interdigitated electrode that was functionalized with Au nanoparticles and the silica precursor TEOS. The red trace is the normalized change in signal from an interdigitated electrode that was functionalized with $\mathrm{Au}$ nanoparticles cross-linked with $\mathbf{1}$ and the silica precursor TEOS. The 1-crosslinked nanoparticle-functionalized device was more sensitive to the same concentration of DMMP, as reflected by the eight-fold increase in detector response.

Figure 3 shows the FID response to the same $1000 \mathrm{ppm}$ DMMP injection both with and without the custom-made test fixture in line with the FID. The peak width of the
$\mathrm{CS}_{2}$ is not dramatically widened with the test fixture in line; however, the DMMP peak appears never to reach the FID detector. This could be evidence of the gettering ability of the assembled film. Based on this evidence, part of the film selectivity to DMMP could arise from the silica matrix surrounding the Au NPs.

The normalized signal response to octane, toluene, and DMMP at various concentrations is shown in Figure 4 and was used to determine the sensitivity of the electrode sensor [13]. Sensitivity is usually defined as the slope of the output signal for a given set of concentrations; as such sensitivity of the assembled sensor was determined for octane $\left(8.0 \mathrm{e}^{-4} \%\right.$ change/ppm), toluene (1.4 $\mathrm{e}^{-2} \%$ change/ppm), and DMMP $\left(8.0 \mathrm{e}^{-1} \%\right.$ change/ppm) between the range of $100 \mathrm{ppm}$ and 50000 ppm.

The limits of detection (LoD) for octane (190 ppm in $\mathrm{CS}_{2}$ ), toluene (80 ppm in $\mathrm{CS}_{2}$ ), and DMMP (60 ppm in $\mathrm{CS}_{2}$ ) were determined from a plot of the log normalized signal change versus log concentration. Based on this plot, a simple extrapolation was used in combination with an arbitrary signal to noise ratio of five (corresponding to $10 \%$ noise) to determine the LoD. In practice, these LoD values were not realized in our experiments likely due to a nonoptimized test cell configuration that leads to band broadening and sensitivity loss. No effort was made to reduce the dead volume of the sensor cell.

The nanoparticles used in the sensing matrix had an average radius size of $4-6 \mathrm{~nm}$, and no attempt was made to synthesize nanoparticles of different sizes or of different 
metals. Presumably, the electron transport properties of the sensing film could be tailored with modifications of both the metal as well as the size of the nanoparticles and will be the subject of future work.

3.3. Role of the Au Nanoparticle to Silica Ratio. Several experiments were performed where the $\mathrm{Au}$ nanoparticle to TEOS ratio was modified, and the IDEs were evaluated upon exposure to DMMP, as shown in Figure 5. Experiments with large $\mathrm{Au}$ nanoparticle:TEOS ratios show results consistent with a conduction mechanism dominated by the first exponential factor of the activated tunneling model. Accordingly, the conductivity was decreased in the presence of both the DMMP $(k=22.3)$ and the solvent $\mathrm{CS}_{2}(k=$ 2.6) [28]. Experiments with small Au nanoparticle:TEOS ratios demonstrated results consistent with a conduction mechanism dominated by the second exponential factor of the activated tunneling model, and showed an increase in conductivity for DMMP. The sample prepared with $0.31 \mathrm{mg}$ $\mathrm{Au} / 10 \mathrm{~mL}$ TEOS exhibited the most sensitivity to DMMP, whereas the smallest $\mathrm{Au}$ nanoparticle:TEOS ratio $(0.16 \mathrm{mg}$ $\mathrm{Au} / 10 \mathrm{~mL}$ TEOS) was not as sensitive. In the latter case, the sensitivity to DMMP is attributed to slow diffusion of the analyte into the sensing film during this nonequilibrium measurement, due to the highly cross-linked nature of the sensing matrix.

\section{Conclusion}

Interdigitated electrodes were functionalized by a flood coating technique, using dodecylamine-capped Au nanoparticles cross-linked with a phenylene ethynylene oligomer in a silica matrix. Control of the nanoparticle:TEOS ratio enables control over which factor dominates in the activated tunneling model. Sensors prepared with $0.31 \mathrm{mg} \mathrm{Au}$ nanoparticles: $10 \mu \mathrm{L}$ TEOS exhibited the greatest increase in conductance upon exposure to DMMP, whereas sensors prepared with $1.25 \mathrm{mg}$ Au nanoparticles:10 $\mu \mathrm{L}$ TEOS showed decreases in conductivity upon exposure to DMMP. Devices functionalized with $0.31 \mathrm{mg}$ Au nanoparticles: $10 \mu \mathrm{L}$ TEOS were 1000 times more sensitive to DMMP than to octane.

\section{Acknowledgments}

Sandia is a multiprogram laboratory operated by Sandia Corporation, a Lockheed Martin Company, for the US Department of Energy under Contract DE-AC04-94AL8500. The same Department of Energy contract supported the internship of BKP when working at Sandia, while her work at Rice University was supported by the Defense Advanced Research Projects Agency.

\section{References}

[1] T. Gao, M. D. Woodka, B. S. Brunschwig, and N. S. Lewis, "Chemiresistors for array-based vapor sensing using composites of carbon black with low volatility organic molecules," Chemistry of Materials, vol. 18, no. 22, pp. 5193-5202, 2006.
[2] K.-S. Teh and L. Lin, "MEMS sensor material based on polypyrrole-carbon nanotube nanocomposite: film deposition and characterization," Journal of Micromechanics and Microengineering, vol. 15, no. 11, pp. 2019-2027, 2005.

[3] J. K. Abraham, B. Philip, A. Witchurch, V. K. Varadan, and C. C. Reddy, "A compact wireless gas sensor using a carbon nanotube/PMMA thin film chemiresistor," Smart Materials and Structures, vol. 13, no. 5, pp. 1045-1049, 2004.

[4] K. Cattanach, R. D. Kulkarni, M. Kozlov, and S. K. Manohar, "Flexible carbon nanotube sensors for nerve agent simulants," Nanotechnology, vol. 17, no. 16, pp. 4123-4128, 2006.

[5] C.-Y. Yang, C.-L. Li, and C.-J. Lu, "A vapor selectivity study of microsensor arrays employing various functionalized ligand protected gold nanoclusters," Analytica Chimica Acta, vol. 565, no. 1, pp. 17-26, 2006.

[6] T. Vossmeyer, B. Guse, I. Besnard, R. E. Bauer, K. Müllen, and A. Yasuda, "Gold nanoparticle/polyphenylene dendrimer composite films: preparation and vapor-sensing properties," Advanced Materials, vol. 14, no. 3, pp. 238-242, 2002.

[7] P. Pang, J. Guo, S. Wu, and Q. Cai, "Humidity effect on the dithiol-linked gold nanoparticles interfaced chemiresistor sensor for VOCs analysis," Sensors and Actuators B, vol. 114, no. 2, pp. 799-803, 2006.

[8] H. Lei and W. G. Pitt, "Selection of polymeric sensor arrays for quantitative analysis," Sensors and Actuators B, vol. 120, no. 2, pp. 386-391, 2007.

[9] N. Krasteva, I. Besnard, Y. Joseph, R. Krustev, A. Yasuda, and T. Vossmeyer, "Analyte sorption and distribution profiles in thin metal nanoparticle/organic composite films-implication to the mechanism of vapor sensing," Chemical Sensors, vol. 20, supplement B, pp. 278-279, 2004.

[10] N. Krasteva, B. Guse, I. Besnard, A. Yasuda, and T. Vossmeyer, "Gold nanoparticle/PPI-dendrimer based chemiresistorsvapor-sensing properties as a function of the dendrimer size," Sensors and Actuators B, vol. 92, no. 1-2, pp. 137-143, 2003.

[11] Y. Joseph, B. Guse, A. Yasuda, and T. Vossmeyer, "Chemiresistor coatings from Pt- and Au-nanoparticle/nonanedithiol films: sensitivity to gases and solvent vapors," Sensors and Actuators B, vol. 98, no. 2-3, pp. 188-195, 2004.

[12] J. Guo, P. Pang, and Q. Cai, "Effect of trace residual ionic impurities on the response of chemiresistor sensors with dithiol-linked monolayer-protected gold (nano)clusters as sensing interfaces," Sensors and Actuators B, vol. 120, no. 2, pp. 521-528, 2007.

[13] M. E. Franke, T. J. Koplin, and U. Simon, "Metal and metal oxide nanoparticles in chemiresistors: does the nanoscale matter?" Small, vol. 2, no. 1, pp. 36-50, 2006.

[14] N. Cioffi, L. Traversa, N. Ditaranto, et al., "Core-shell Pd nanoparticles embedded in SnOx films. Synthesis, analytical characterisation and perspective application in chemiresistortype sensing devices," Microelectronics Journal, vol. 37, no. 12, pp. 1620-1628, 2006.

[15] Y. Joseph, N. Krasteva, I. Besnard, et al., "Goldnanoparticle/organic linker films: self-assembly, electronic and structural characterisation, composition and vapour sensitivity," Faraday Discussions, vol. 125, pp. 77-97, 2004.

[16] W. P. Wuelfing, S. J. Green, J. J. Pietron, D. E. Cliffel, and R. W. Murray, "Electronic conductivity of solid-state, mixed-valent, monolayer-protected Au clusters," Journal of the American Chemical Society, vol. 122, no. 46, pp. 11465-11472, 2000.

[17] H. F. Xiang, H. Y. Xu, Z. Z. Wang, and C. H. Chen, "Dimethyl methylphosphonate (DMMP) as an efficient flame retardant 
additive for the lithium-ion battery electrolytes," Journal of Power Sources, vol. 173, no. 1, pp. 562-564, 2007.

[18] M. Brust, M. Walker, D. Bethell, D. J. Schiffrin, and R. Whyman, "Synthesis of thiol-derivatised gold nanoparticles in a two-phase Liquid-Liquid system," Journal of the Chemical Society, Chemical Communications, vol. 7, pp. 801-802, 1994.

[19] H. Fan, K. Yang, D. M. Boye, et al., "Self-assembly of ordered, robust, three-dimensional gold nanocrystal/silica arrays," Science, vol. 304, no. 5670, pp. 567-571, 2004.

[20] K. Sonogashira, Y. Tohda, and N. Hagihara, "Convenient synthesis of acetylenes: catalytic substitutions of acetylenic hydrogen with bromo alkenes, iodo arenes, and bromopyridines," Tetrahedron Letters, vol. 16, no. 50, pp. 4467-4470, 1975.

[21] C. Cai and A. Vasella, "Oligosaccharide analogues of polysaccharides-part 3. A new protecting group for alkynes: orthogonally protected dialkynes," Helvetica Chimica Acta, vol. 78, no. 3, pp. 732-757, 1995.

[22] G. J. Bodwell, D. O. Miller, and R. J. Vermeij, "Nonplanar aromatic compounds. 6. [2] paracyclo[2] (2,7)pyrenophane. A novel strained cyclophane and a first step on the road to a "Vögtle" Belt," Organic Letters, vol. 3, no. 13, pp. 2093-2096, 2001.

[23] J. Wu, C. Chi, X. Wang, J. Li, X. Zhao, and F. Wang, "A one-pot procedure to prepare S-protected 4-iodothiophenols," Synthetic Communications, vol. 30, no. 23, pp. 4293-4298, 2000.

[24] N. Stuhr-Hansen, J. K. Sørensen, K. Moth-Poulsen, J. B. Christensen, T. Bjørnholm, and M. B. Nielsen, "Synthetic protocols and building blocks for molecular electronics," Tetrahedron, vol. 61, no. 52, pp. 12288-12295, 2005.

[25] J. M. Tour, L. Jones II, D. L. Pearson, et al., "Self-assembled monolayers and multilayers of conjugated thiols, $\alpha, \omega$-dithiols, and thioacetyl-containing adsorbates. Understanding attachments between potential molecular wires and gold surfaces," Journal of the American Chemical Society, vol. 117, no. 37, pp. 9529-9534, 1995.

[26] L. Cai, Y. Yao, J. Yang, D. W. Price Jr., and J. M. Tour, "Chemical and potential-assisted assembly of thiolacetylterminated oligo(phenylene ethynylene)s on gold surfaces," Chemistry of Materials, vol. 14, no. 7, pp. 2905-2909, 2002.

[27] A. Shaporenko, M. Elbing, A. Blaszczyk, C. von Hänisch, M. Mayor, and M. Zharnikov, "Self-assembled monolayers from biphenyldithiol derivatives: optimization of the deprotection procedure and effect of the molecular conformation," Physical Chemistry B, vol. 110, no. 9, pp. 4307-4317, 2006.

[28] M. G. Gaikwad, S. Deuskar, and S. K. David, "Study of dependence of dielectric constant of liquids on free volume," Solid State Communications, vol. 39, no. 1, pp. 65-69, 1981. 

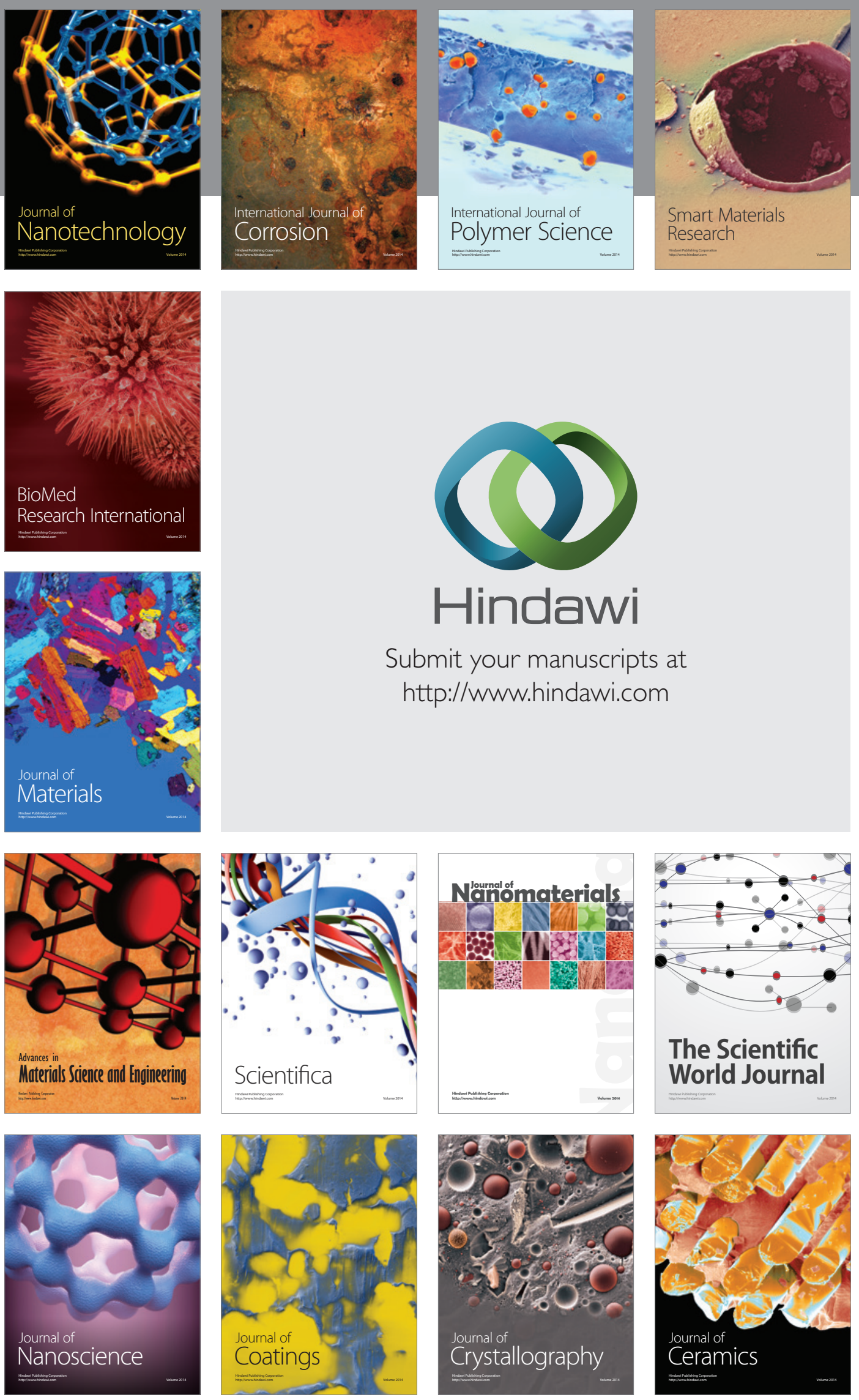

The Scientific World Journal

Submit your manuscripts at

http://www.hindawi.com

\section{World Journal}

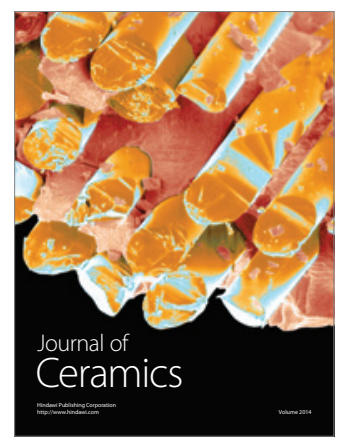

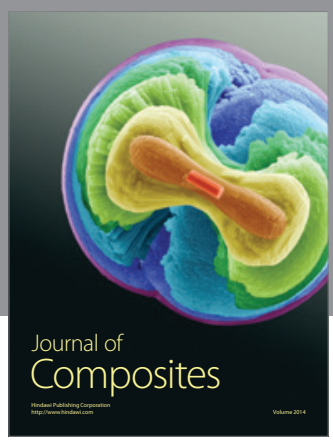
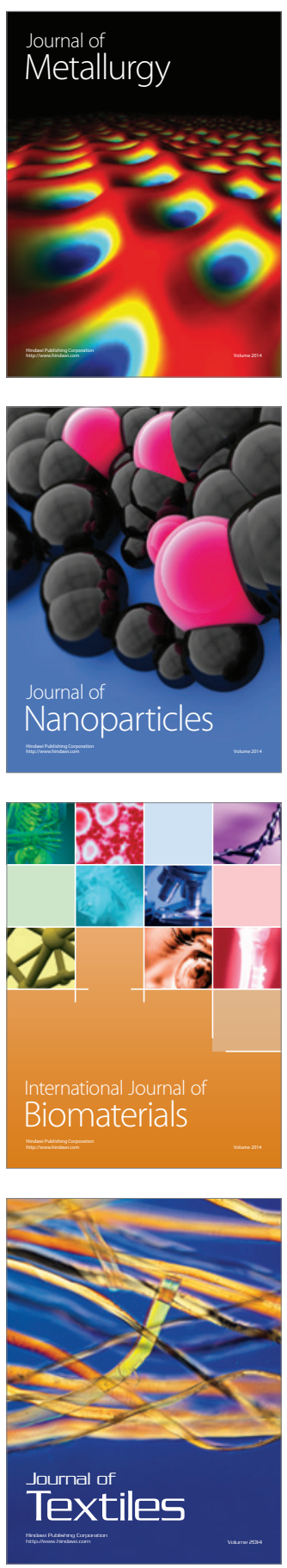